\title{
Capillary Hydraulic Jump in a Viscous Jet
}

\author{
A. A. Safronov, A. A. Koroteev, N. I. Filatov, N. A. Safronova
}

Stationary waves in a cylindrical jet of a viscous fluid are considered. It is shown that when the capillary pressure gradient of the term with the third derivative of the jet radius in the axial coordinate is taken into account in the expression, the previously described self-similar solutions of hydrodynamic equations arise. Solutions of the equation of stationary waves propagation are studied analytically. The form of stationary soliton-like solutions is calculated numerically. The results obtained are used to analyze the process of thinning and rupture of jets of viscous liquids.

Keywords: instability, capillary flows, viscous jet, stationary waves

Received April 29, 2019

Accepted July 09, 2019

This work was supported by the grant of the Russian Science Foundation No. 19-19-00045.

Andrey A. Safronov

a.a.safr@yandex.ru

Keldysh Research Center

ul. Onezhskaya 8, Moscow, 125438 Russia

Anatoly A. Koroteev

chkt@yandex.ru

Moscow Aviation Institute (National Research University)

Volokolamskoe sh. 4, Moscow, 125993 Russia

Nikolay I. Filatov

chkt@yandex.ru

Keldysh Research Center

ul. Onezhskaya 8, Moscow, 125438 Russia

Nadezhda A. Safronova

safronova.na@list.ru

Moscow Institute of Physics and Technology

Institutsky per. 9, Dolgoprudny, Moscow region, 141701 Russia 


\section{Introduction}

Modeling the capillary breakup of viscous fluid jets is an important task for many technical applications $[1-8,12-16]$. One of them is a liquid droplet radiator designed to remove low-grade heat from new-generation space power systems. The idea of droplet radiator consists in the radiation cooling of specially formed dispersed flow, which freely propagates in space [1]. The requirement of low evaporability is placed on the heat carrier of such radiators. All potentially suitable working fluids for use in space have sufficiently high viscosity. The regularities of the forced capillary disintegration of jets of such liquids were considered in [2], where the dependence of the sizes of the main and satellite drops on the wave number of the initiating decay of the perturbation at different viscosity values was obtained. However, the results of experimental studies indicate that the capillary disintegration of highly viscous jets may be accompanied by the formation of not only satellite drops, but also subsatellites of small size [3-5]. One main drop may account for several subsatellites.

Figure 1 shows photographs of the process of the formation of subsatellites in the gap between the pre-main drop and pre-satellite drop during the disintegration of the jet of vacuum oil BM1-C. An experimental study of the subsatellites formation is difficult by the small scale of the processes taking place: it is necessary to measure the radius of the decaying jet with an error of less than $\sim 10^{-6} \mathrm{~m}$ with a frequency of approximately $1 \mathrm{MHz}$. Therefore, mathematical modeling is practically the only way to study the regularity of formation of subsatellites.

An analytical model of the formation of pre-subsatellites in a decaying jet of a highly viscous fluid was proposed in [6], based on the description of the development of hydrodynamic instabilities of capillary flow in thin constriction connecting the pre-drops. The formation of pre-drop is associated with the multistability of solving the equation of thinning the constriction, and their shape is described by solving the nonlinear differential equation. The results of numerical calculations show that an initially regular structure of the waves, close to the analytically calculated one, is formed in the constriction. Thus, there is a complication of the structure and the loss of its regularity.

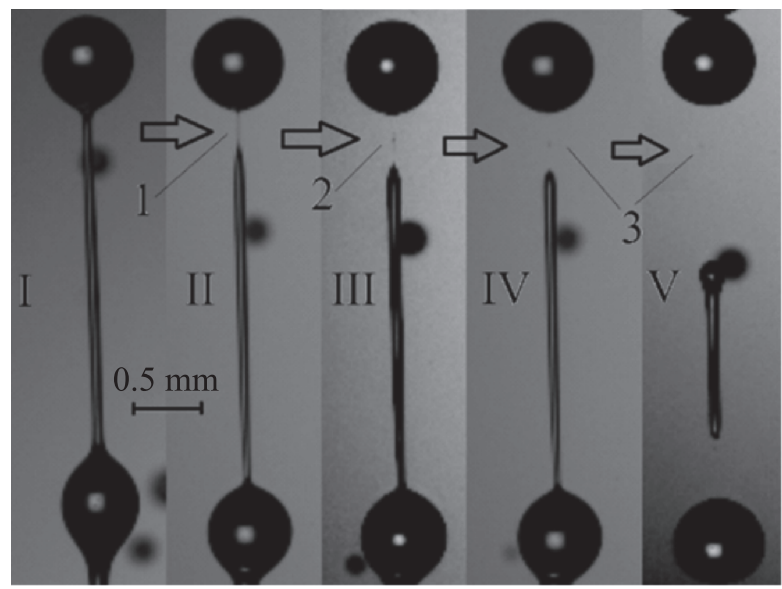

Fig. 1. Consecutive photographs of the process of formation of subsatellite drops in the constriction connecting pre-main drop and pre-satellite drop. Time: I - initial moment of time, $0 \mu \mathrm{s}, \mathrm{II}-2300 \mu \mathrm{s}$, III - $3200 \mu \mathrm{s}, \mathrm{IV}-3400 \mu \mathrm{s}, \mathrm{V}-7200 \mu \mathrm{s}$. The numbers denote: 1 - constriction connecting the pre-main drop and pre-satellite drop; 2 - pre-subsatellite; 3 - subsatellites. 
In the present work, we investigate the phenomenon of hydraulic jump in a jet of a viscous fluid, which is one of the components of the process of destruction of the regular wave structure of the subsystems of subsatellites.

\section{Equations of evolution of capillary disturbances in a jet}

The capillary breakup of a cylindrically symmetric jet of a viscous Newtonian fluid was simulated. The calculation of the evolution of the wave structure of pre-subsatellites in the jet was carried out in cylindrical coordinates, the axis of which coincides with the axis of the jet, in dimensionless variables. For the dimensioning of the jet radius $r$, the velocity of fluid $v$ and time $\widetilde{t}$ were used: the radius of the unperturbed jet $r_{0}: r=\frac{\widetilde{r}}{r_{0}} ;$ the velocity $v_{0}=\sqrt{\frac{\sigma}{\rho r_{0}}}(\sigma$ and $\rho$ are the surface tension and the fluid density, respectively): $u=\frac{\widetilde{v}}{v_{0}} ;$ and time $t_{0}=\sqrt{\frac{\rho r_{0}^{3}}{\sigma}}$ : $t=\frac{\widetilde{t}}{t_{0}}$. To describe the effect of viscosity, the Ohnesorge number was used, which is an analogue of the Reynolds number for capillary waves in the jet, determined using the ratio $\mathrm{Oh}=\frac{\mu}{\sqrt{\sigma \rho r_{0}}}$, where $\mu$ is the dynamic viscosity of the fluid.

In the approximation of long (compared with the jet radius) waves, the dimensionless hydrodynamic system of equations is written in the form

$$
\begin{gathered}
\partial_{t} u+u \partial_{x} u=-\partial_{x} \mathfrak{L}+3 \mathrm{Oh} \frac{1}{r^{2}} \partial_{x}\left(r^{2} \partial_{x} u\right), \\
\partial_{t} r+u \partial_{x} r=-\frac{1}{2} r \partial_{x} u
\end{gathered}
$$

where function $\mathfrak{L}$ describes the dependence of the curvature of the jet surface on the coordinate. The derivation of Eqs. (2.1)-(2.2) from the Navier - Stokes system is given in [7].

The dynamic equation (2.1) expresses the momentum conservation law. The left-hand side of this equation contains the convective derivative of the velocity of jet substance. The term $\mathfrak{L}$ expresses the action of capillary forces. The term of the equation which contains the Ohnesorge number expresses the action of viscous friction forces. The formula (2.2) is a continuity equation.

The present work is focused on phenomena which accompany the process of capillary breakup of jets. The experiment (see Fig. 1) shows that jet breakup occurs in two steps. At the first step pre-drops are formed. These pre-drops are connected with thin liquid threads (Fig. 1-I, Fig. 1-II). At the second step the thin liquid threads break (Fig. 1-II-Fig. 1-IV). It is known that the breakup of this threads can be described with patterns which are close to self-similar [8]. However, there are quite a lot of different suitable patterns. The pattern of choice depends on the ratio of inertia, friction, and surface tension forces.

When the Ohnesorge number of breaking jet is large enough, the inertia influence on the thread breakup is negligible. The flow dynamics is determined by surface tension and viscosity. As a result, the so-called Stokes self-similar solution takes place [9]. This solution is marked by symmetrical dependence $r(x)$ relative to the point of jet breakup $x^{*}$. The dependence is close to parabolic. In the case where inertia, viscosity and surface tension forces are comparable, the so-called universal self-similar Navies - Stokes solution takes place [9]. In this case the jet shape is asymmetrical relative to the breakup point. 
In a number of papers (one can find references in detailed reviews $[8,9]$ ) these self-similar solutions were explored theoretically, where in order to simplify the solution, the following approximate equation of dependence of the curvature of the jet surface was used:

$$
\mathfrak{L}=\frac{1}{r} .
$$

In [10], the research on stability of universal self-similar Navies - Stokes solution is published. The shape of a fluid interface undergoes repeated instabilities arbitrarily close to breakoff. This behavior is the result of successive instabilities of the similarity solution. The similarity solution is unstable to finite amplitude perturbations, with critical amplitude going to zero at the singularity.

The stability analysis of the self-similar Stokes solution [11], carried out with the value $\mathfrak{L}$ calculated by Eq. (2.3), shows that there exists an infinite hierarchy of similarity solutions corresponding to pinchoff. Only one of the similarity solutions (the "ground state") is stable, all other solutions are linearly unstable to perturbations, and thus cannot be observed.

In [5], instabilities of the self-similar Stokes solution were observed in the experiments on the capillary jet breakup of highly viscous liquids. Those instabilities could not be explained by the theory proposed in [11]. However, the instabilities led to the formation of subsatellites. One can describe the evolution of the instability by using the equation for dependence of the curvature of the jet surface which is more precise than (2.3). This equation is the following:

$$
\mathfrak{L}=\frac{1}{r}-\partial_{x x} r .
$$

The breakup of the thread in between the pre-drops is characterized by complicated dynamics [5]. Firstly, nonlinear waves form at the well-formed self-similar Stokes solution. Secondly, these waves interact with each other. This interaction leads to the loss of regularity in wave structure. At the final step of jet breakup the area of self-similar jet constriction can form. The patterns of capillary flow in this area are different from both self-similar Navies - Stokes and Stokes solutions.

A feature of the system of the development of capillary perturbations in the jet (2.1), (2.2), (2.4) investigated in this work is the inclusion of the second derivative of the jet radius in the curvature value of the jet surface $\mathfrak{L}$. Because of this, when calculating the capillary pressure gradient $\partial_{x} \mathfrak{L}$ in Eq. (2.1), a term with the third derivative of the jet radius $\partial_{x x x} r$ appears. Self-similar solutions for jet refinement were described in a simplified case in [8], when $\mathfrak{L}=\frac{1}{r}$. However, when the value of the Ohnesorge number is close to one, the inclusion of the value $\partial_{x x x} r$ in Eq. (2.1) destroys these self-similar solutions and generates new self-similar solutions.

\section{Numerical simulation of the evolution of the wave structure}

The numerical solution of the system of Eqs. (2.1), (2.2), (2.4) was carried out by the finite difference method on an eight-point implicit scheme (for more on this, see [5]). Below, when presenting the results, the time was counted from the moment of jet break $t^{*}$. The value of the Ohnesorge number was assumed to be 0.75 , and the wave number of the initiating capillary disruption of the perturbation was taken to be $k=\frac{2 \pi r_{0}}{\lambda}=0.2$ ( $\lambda$ being the perturbation wave length). The initial condition for the jet radius was chosen in the standard form for the problem of simulating a forced capillary breakup:

$$
r(x)=1+\varepsilon \cos (k x)-\frac{\varepsilon^{2}}{4},
$$


where $\varepsilon$ is the dimensionless amplitude of the perturbation, which was taken to be 0.05 . The term of the equation containing the square of $\varepsilon$ is introduced to keep the volume of the fluid constant. The velocity of the substance in the jet at the initial moment of time was taken to be zero. The calculation was carried out on the interval $x \in[-\lambda / 2, \lambda / 2]$.

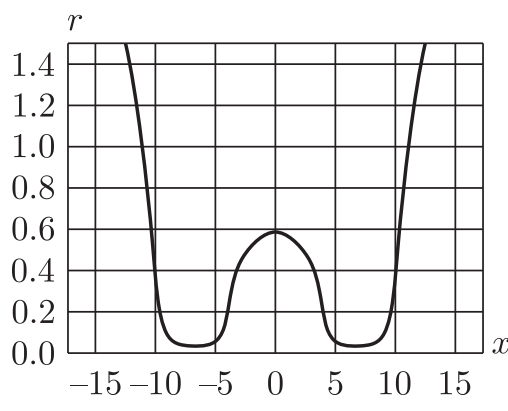

a)

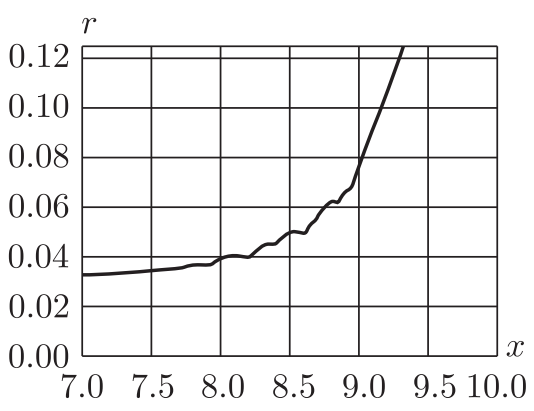

b)

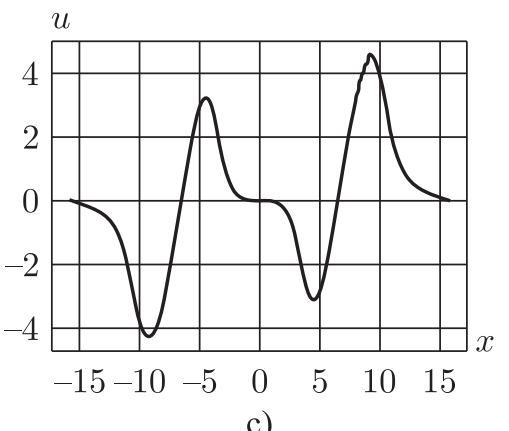

c)

Fig. 2. Dependences a) and b) on the jet radius on the coordinate at different scales and c) the velocity of the substance in it on the coordinate at the moment of formation of pre-subsatellites $(t *-t)=0.42$.
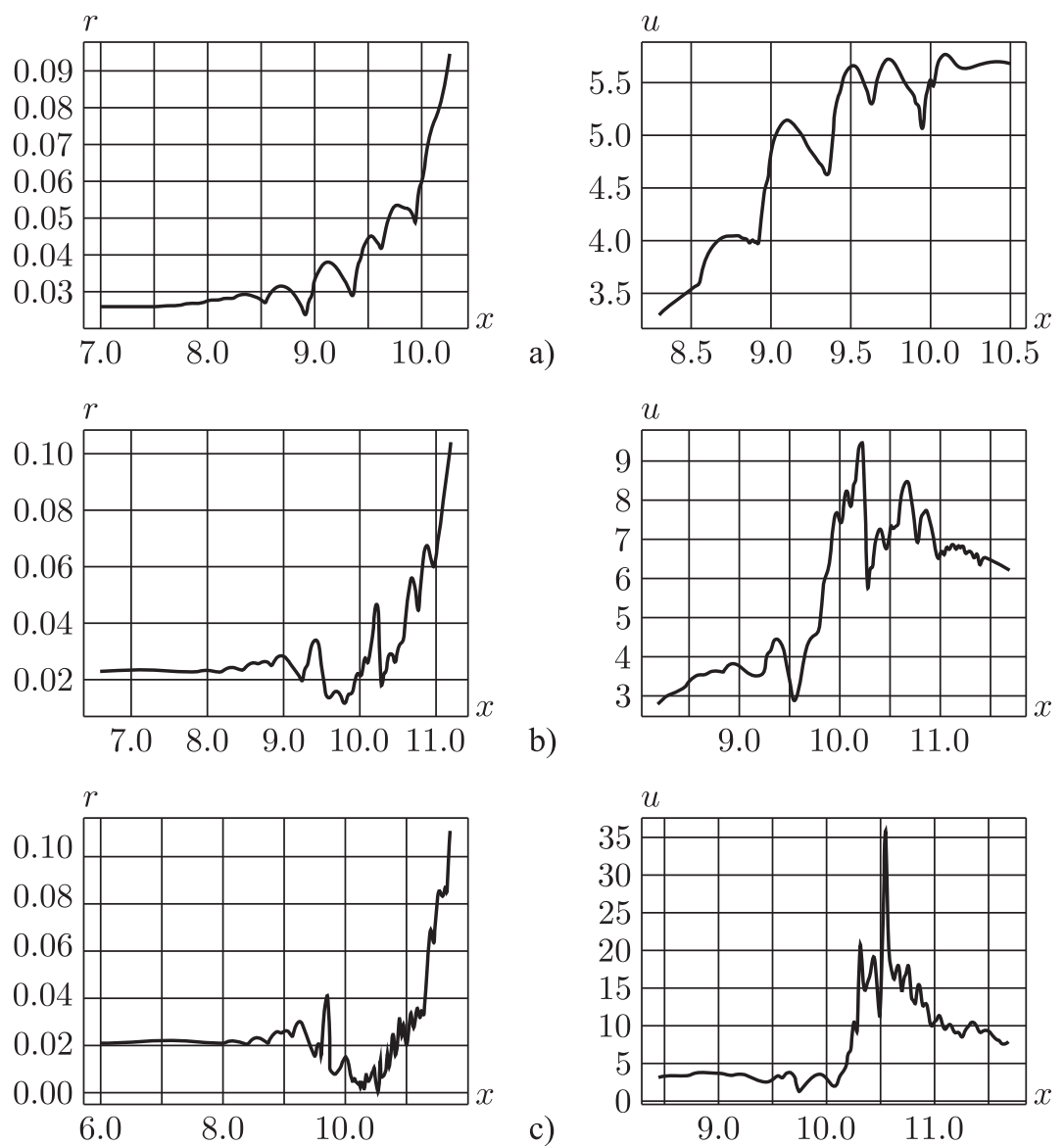

Fig. 3. The evolution of the wave structure over time: a) $(t *-t)=0.2065 ; \mathrm{b})(t *-t)=0.0636$; c) $(t *-t)=0.0003$. 
The wave structure of pre-subsatellites is formed in the vicinity of the connection of the thin constriction and the pre-drop. Figure 2 shows the dependence of the jet radius on the coordinate and velocity of the substance after the formation of pre-drops. Figure 2c shows that initially the wave structure has little effect on the jet velocity field.

Figure 3 shows the results of calculations of the evolution of the wave structure of presubsatellites over time. As the amplitudes of the waves grow, on their crests and in the intervals between them, growing secondary waves are formed (Fig. 3b). Before the moment of jet break, it is possible to complete several such iterations, which lead to the randomization of the wave structure (Fig. 3c). It can be seen from the figures that in the region of loss by wave structure of regularity, a disturbance similar to the hydraulic jump is formed.

In numerical simulation, a slow evolution of the formed wave structures was observed. In the first approximation, they can be described as stationary waves.

\section{A stationary wave in a jet of a viscous fluid}

It was considered that a stationary wave with velocity $c$ propagated in the jet. The velocity of the fluid before the wave front is equal to $-v$ (see Fig. 4). The jet radius $r \rightarrow 1$ as $x \rightarrow+\infty$.

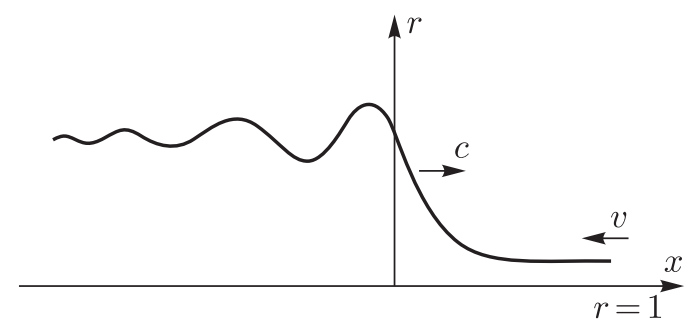

Fig. 4. The characteristic form of the profile of a stationary wave.

The dependence of the jet radius on the coordinate in the stationary wave was represented as $r(x, t)=r(x-c t)=r(\xi)$, where $\xi=x-c t$. Equations (2.1), (2.2) and (2.4) take the form

$$
\begin{gathered}
u^{\prime}(u-c)=\frac{r^{\prime}}{r^{2}}+r^{\prime \prime \prime}+3 \mathrm{Oh} u^{\prime \prime}+6 \mathrm{Oh} u^{\prime} \frac{r^{\prime}}{r}, \\
r^{\prime}(u-c)=-\frac{1}{2} r u^{\prime} .
\end{gathered}
$$

Considering $\alpha=c+v$, after integrating Eq. (2.5) we get

$$
u-c=-\frac{\alpha}{r^{2}}
$$

Using (2.6), Eq. (2.4) can be converted to the form

$$
r^{\prime \prime \prime}+\frac{r^{\prime}}{r^{2}}\left(1+\frac{2 \alpha^{2}}{r^{3}}\right)+6 \mathrm{Oh} \frac{\alpha}{r^{4}}\left[\frac{1}{2}\left(r^{2}\right)^{\prime \prime}-2 r^{\prime 2}\right]=0 .
$$

Equation (2.7) satisfies any solution of the form $r=$ const. However, there is special class of such solutions, which are attractors for moving trajectories of the dynamical system (2.7). 
For a qualitative analysis of the behavior of stationary waves in the viscous jet, we can neglect in (2.4) the term $6 \mathrm{Oh} u^{\prime} r^{\prime} / r$. From relation (2.7) it can be seen that, under this assumption, there is a slight change in the decay rate of the oscillations. Then

$$
r^{\prime \prime \prime}+\frac{r^{\prime}}{r^{2}}\left(1+\frac{2 \alpha^{2}}{r^{3}}\right)+3 \mathrm{Oh} u^{\prime \prime}=0
$$

Taking the ratios (2.6) into account, integrating the resulting relation with respect to $\xi$ yields the expression

where $C_{1}=$ const.

$$
r^{\prime \prime}-\frac{\alpha^{2}}{2 r^{4}}-\frac{1}{r}+6 \mathrm{Oh} \frac{\alpha}{r^{3}} r^{\prime}+C_{1}=0
$$

Considering that $r^{\prime}, r^{\prime \prime} \rightarrow 0, r \rightarrow 1$ as $\xi \rightarrow+\infty$, we obtain

$$
C_{1}=\frac{1}{2} \alpha^{2}-1
$$

Considering (3.1), Eq. (2.9) takes the form

$$
r^{\prime \prime}+6 \mathrm{Oh} \frac{\alpha}{r^{3}} r^{\prime}-\frac{\alpha^{2}}{2 r^{4}}-\frac{1}{r}+\frac{1}{2} \alpha^{2}-1=0 .
$$

The last relation describes the motion of a damped oscillator in the potential

$$
V(r)=\frac{\alpha^{2}}{6 r^{3}}-\ln (r)+\left(\frac{1}{2} \alpha^{2}-1\right) r .
$$

The potential $V$, defined by (3.3), has a minimum in the vicinity of which the system's trajectory oscillates as $\alpha>\alpha^{*} \approx 1.415$. Figure 5 shows the characteristic form of the potential $V(r)$, when it has a minimum, as well as the dependence of the position of the minimum on the value of $\alpha$. For large values of $\alpha$, the increase in the jet radius when the disturbance passes is small. But when the value of $\alpha$ is close to the critical $\alpha^{*}$, the jet radius can change several times. With large changes in the radius, a situation may arise in which one wave front oscillates and the other monotonously decreases. Note that the assumptions used in deriving relation (3.3) do not affect the equilibrium position of the system, but only affect the speed at which it is approached.

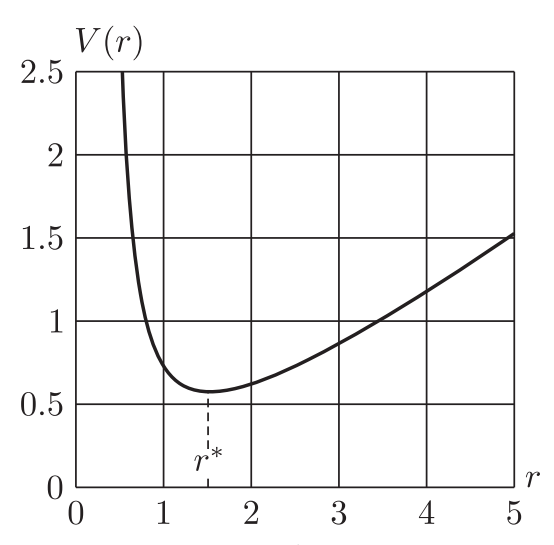

a)

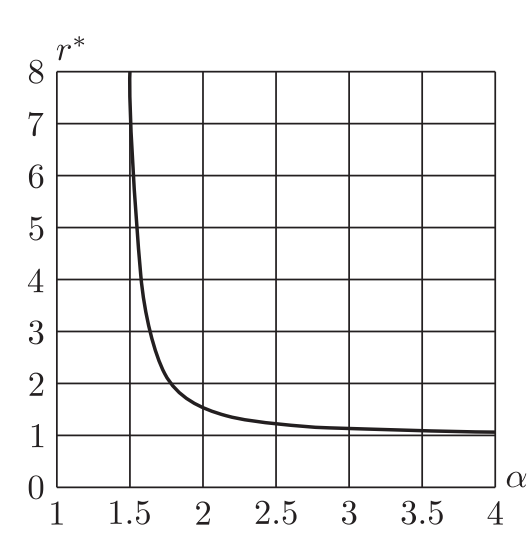

b)

Fig. 5. a) $V(r)$ change at $\alpha=1.7$ ( $r^{*}$ is the equilibrium position); b) the dependence of $r^{*}$ on $\alpha$. 
The results of the analysis of Eq. (2.8), which approximately describes the solutions (2.7), indicate that there are solutions of model equations such as hydraulic jumps, which have the following asymptotics: as $\xi \rightarrow-\infty, r \rightarrow 1 ; r^{\prime}, r^{\prime \prime} \rightarrow 0$; and as $\xi \rightarrow+\infty, r \rightarrow r^{*} ; r^{\prime}, r^{\prime \prime} \rightarrow 0$.

A study of the stability loss of the stationary solution $r=1$ of Eq. (2.7) was conducted. The dependence $r(\xi)$ was represented as $r=1+p(\xi)$, where $p$ is a small deviation. In the linear approximation of the expansion in $p$, Eq. (2.7) takes the form

$$
p^{\prime \prime \prime}+6 \mathrm{Oh} \alpha p^{\prime \prime}+p^{\prime}\left(2 \alpha^{2}+1\right)=0 .
$$

Considering that $p=p_{0} \exp (\xi)$, it is possible to get the equation

$$
\omega\left(\omega^{2}+6 \mathrm{Oh} \alpha \omega+1+2 \alpha^{2}\right)=0 .
$$

This equation has three roots: $\omega_{1}=0$ and

$$
\omega_{2,3}=-3 \mathrm{Oh} \alpha\left(1 \pm \sqrt{1-\frac{1+2 \alpha^{2}}{9 O h^{2} \alpha^{2}}}\right) .
$$

Thus, Eq. (3.4) has only nonincreasing solutions.

If $\mathrm{Oh}<\frac{\sqrt{1+2 \alpha^{2}}}{3 \alpha}$, oscillating solutions arise in the system with wave number $L$ equal to

$$
L=\sqrt{1+2 \alpha^{2}-9 \mathrm{Oh}^{2} \alpha^{2}} .
$$

When $\mathrm{Oh}<\sqrt{2} / 3$, the function $L$ increases with an increase in the absolute value of $\alpha$. For large values of the Ohnesorge number, the wave number decreases with increasing modulus $\alpha$. Figure 6 shows the dependence of $L$ on $\alpha$ for large $(\mathrm{Oh}=0.7)$ and small $(\mathrm{Oh}=0.1)$ values of the Ohnesorge number.

Note that, as $\alpha \rightarrow \infty$, not only the wave number of disturbances, but also the damping decrement of such waves increases: at wave length, their amplitude decreases by $\sim e^{2 \pi}$ times.

When $\omega_{1}, \omega_{2}, \omega_{3}$ are real numbers, the solution of Eq. (2.7) may be nonmonotonic. The specific type of solution is determined by the initial conditions. To solve the differential equation (2.7), it is necessary to set three initial conditions that can be interpreted as a jet radius before and after a hydraulic jump, as well as the maximum (if the jump occurs with the formation of a thickening area) or the minimum (in the opposite case) jet radius value.

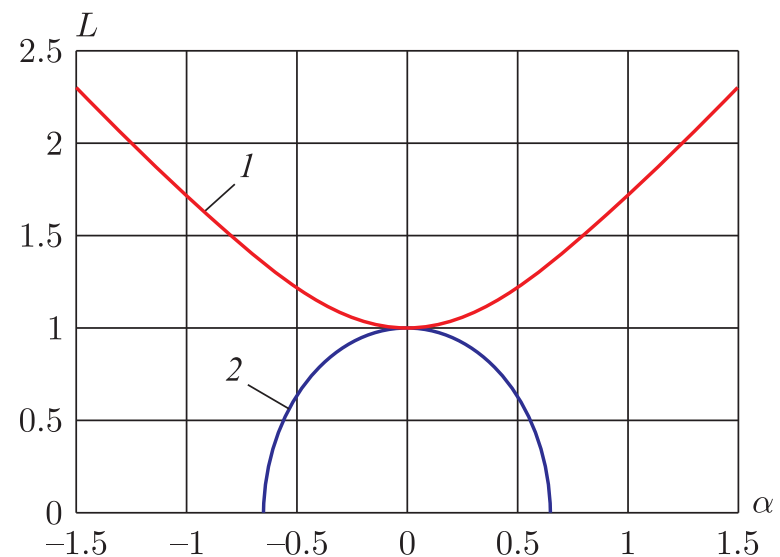

Fig. 6. The dependence of the wave number $L$ on $\alpha(1-\mathrm{Oh}=0.1 ; 2-\mathrm{Oh}=0.7)$. 


\section{Numerical simulation of stationary waves}

The numerical solution of Eq. (2.7) allows one to detect soliton-like structures. Figure 7a shows such a wave, obtained at: $r(0)=1, r^{\prime}(0)=-0.5, r^{\prime \prime}(0)=-2$, Oh $=0.75, \alpha=0.7$. Figure $7 \mathrm{~b}$ shows the dependence of the jet radius on the coordinate at the moment of time $\left(t^{*}-t\right)=0.0003$ (corresponds to Fig. 3c). It can be seen that during the evolution of disturbances in the wave structure of pre-subsatellites, waves similar to the solitary wave shown in Fig. 7a are also formed.
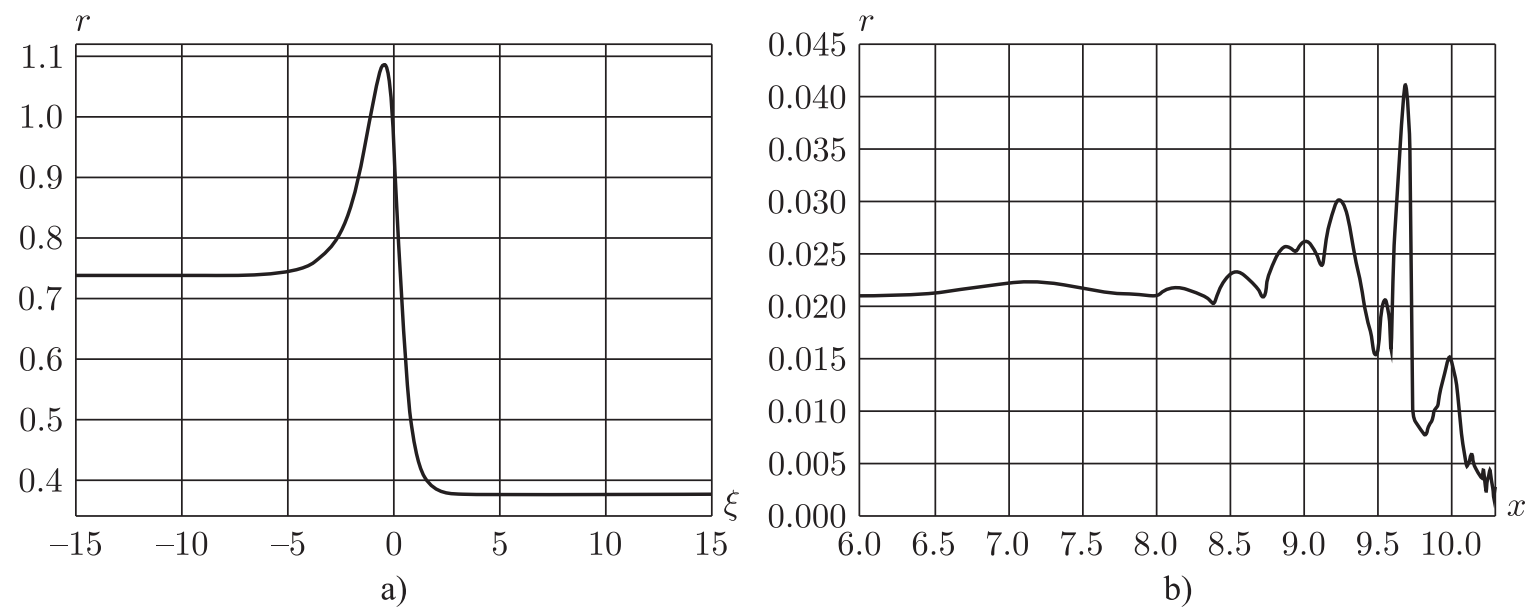

Fig. 7. a) The dependence $r(\xi)$, calculated for $r(0)=1, r^{\prime}(0)=-0.5, r^{\prime \prime}(0)=-2$, Oh $=0.75, \alpha=0.7$; b) decay jet profile at the moment of time $\left(t^{*}-t\right)=0.0003$ (corresponds to Fig. 3c).

Figure 8 shows the solution to Eq. (2.7) at $\mathrm{Oh}=0.75, \alpha=1.5, r(0)=0.815, r^{\prime}(0)=0$, $r^{\prime \prime}(0)=2$. A single wave is being formed; the radius of the jet during the passage of the jump varies slightly.

Relation (2.7) is obtained under the assumption that the curvature of the jet surface varies according to the law $\mathfrak{L}=\frac{1}{r}-\partial_{x x} r$. This approximate relationship is applicable when the disturbance wave length significantly exceeds the jet radius. It follows from (4.1) that in some cases short waves are formed during the propagation of stationary waves in the jet. For their

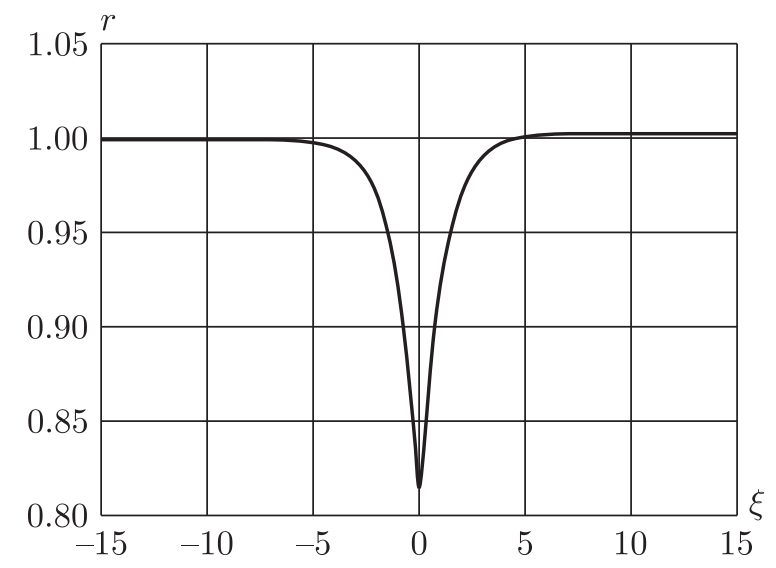

Fig. 8. The dependence $r(\xi)$, calculated for $\mathrm{Oh}=0.75, r(0)=0.815, r^{\prime}(0)=0, r^{\prime \prime}(0)=2, \alpha=1.5$. 
description, we have used the generalized dependence of the curvature of the jet on the radius:

$$
\mathfrak{L}=r^{-1}\left(1+\left(\partial_{x} r\right)^{2}\right)^{-\frac{1}{2}}-\partial_{x x} r\left(1+\left(\partial_{x} r\right)^{2}\right)^{-\frac{3}{2}} .
$$

Then

$$
\begin{aligned}
r^{\prime \prime \prime}\left(1+r^{\prime 2}\right)^{-\frac{3}{2}}-3 r^{\prime} r^{\prime \prime 2}\left(1+r^{\prime 2}\right)^{-\frac{5}{2}}+\frac{r^{\prime}}{r^{2}}\left(1+r^{\prime 2}\right)^{-\frac{1}{2}}+\frac{r^{\prime} r^{\prime \prime}}{r}\left(1+r^{\prime 2}\right)^{-\frac{3}{2}}+ & \\
& +\frac{2 \alpha^{2} r^{\prime}}{r^{5}}+6 \mathrm{Oh} \frac{\alpha}{r^{4}}\left(\frac{1}{2}\left(r^{2}\right)^{\prime \prime}-2 r^{\prime 2}\right)=0 .
\end{aligned}
$$

Comparison of solutions of Eqs. (2.7) and (5.1) reveals their similar structure. At the same time, the perturbation wave lengths obtained by solving Eq. (5.1) are longer wave lengths (the ratio can reach 1.5).

\section{Discussion of the results}

It follows from (4.2) that, when $\mathrm{Oh}<\sqrt{2} / 3$, waves with a wave number $k>1$ may appear in the jet. According to the classic Rayleigh results [12], the jet surface is unstable when $k<1$. The explanation of the formation of short waves can be based on the results of $[13,14]$, which show that for $k>1$ multimode surface disturbances with different frequencies and sufficiently high amplitudes increase. Apparently, it is also necessary to take into account the fact that the short waves described in this paper are in essence a dissipative structure that is formed at values of the velocity difference at which the possibility of applying Rayleigh's theory is doubtful.

The results of this work were obtained in the approximation of stationary waves. Previously, a similar approximation had been used to simulate the formation of periodic structures in thinning constrictions of viscoelastic polymeric liquids (for example, [15]).

The study of the effect of capillary forces on the formation of hydraulic jumps was carried out earlier in a number of other publications, for example, in [16, 17]. In [16], the distribution of fluid in a complex region formed by foam bubbles was investigated. A special feature of the flow was the negative curvature of the surface of the liquid, causing the occurrence of a hydraulic jump in it. In [17], an analysis is made of the formation of circular thin-film hydraulic jumps that occur when the jet falls on a flat surface. In the case of small thickness of the propagating film, the action of gravity does not play a significant role in what is happening, and the formation of the hydraulic jump is due to the action of friction and surface tension.

In contrast to the publications listed above, this study shows the theoretical possibility of the existence of a hydraulic jump in a cylindrical jet of a viscous fluid. Mathematically, the formation of the jump is due to fact that the term with the third derivative of the jet radius with respect to the axial coordinate can be present in the expression of the gradient of capillary pressure $\partial_{x} \mathfrak{L}=\partial_{x x x} r-\frac{\partial_{x} r}{r^{2}}$.

\section{References}

[1] Koroteev, A. A., Safronov, A. A., and Filatov, N. I., Influence of the Structure of a Droplet Sheet on the Capacity of Frameless Space Radiators and the Efficiency of the Power Units, High Temp., 2016, vol.54, no.5, pp.767-770; see also: Teplofizika Vysokikh Temperatur, 2016, vol.54, no.5, pp. $817-820$. 
[2] Safronov, A. A., Features of Capillary Breakup of a Liquid Jet at Ohnesorge Numbers Larger Than Unity, J. Eng. Phys. Thermophys., 2017, vol.90, no.1, pp.167-175; see also: Inzh.-Fiz. Zh., 2017, vol. 90, no. 1, pp. 176-185.

[3] Driessen, T., Jeurissen, R., Wijshoff, H., Toschi, F., and Lohse, D., Stability of Viscous Long Liquid Filaments, Phys. Fluids, 2013, vol. 25, no. 6, 062109, 7 pp.

[4] Tjahjadi, M., Ottino, J.M., and Stone, H. A., Satellite and Subsatellite Formation in Capillary Breakup, J. Fluid Mech., 1992, vol. 243, pp. 297-317.

[5] Grigoriev, A. L., Koroteev, A. A., Safronov, A. A., and Filatov, N. I., Self-Similar Patterns of Subsatellites Formation at the Capillary Breakup of Viscous Jets, Thermophys. Aeromech., 2018, vol. 25, no. 4, pp. 575-585.

[6] van der Bos, J. A., van der Meulen, M.P., Driessen, T.W., van den Berg, M., Reinten, H., Wijshoff, M. A., and Lohse, D., Velocity Profile inside Piezoacoustic Inkjet Droplets in Flight: Comparison between Experimental and Numerical Simulation, Phys. Rev. Appl., 2014, vol. 1, no. 1, 014004, 9 pp.

[7] Eggers, J. and Todd, F. D., Drop Formation in a One-Dimensional Approximation of the NavierStokes Equation, J. Fluid Mech., 1997, vol. 262, pp. 205-221.

[8] Eggers, J. and Villermaux, E., Physics of Liquid Jets, Rep. Prog. Phys., 2008, vol. 71, no. 3, 036601, $79 \mathrm{pp}$.

[9] Eggers, J., Drop Formation: An Overview, Z. Angew. Math. Mech., 2005, vol. 85, no. 6, pp. 400-410.

[10] Brenner, M. P., Shi, X. D., and Nagel, S. R., Iterated Instabilities during Droplet Fission, Phys. Rev. Lett., 1994, vol. 73, no. 25, pp. 3391-3394.

[11] Brenner, M.P., Stability of a Viscous Pinching Thread, Phys. Fluids, 2012, vol.24, no. 7, 072103, $11 \mathrm{pp}$.

[12] Strutt, J.W. (3rd Baron Rayleigh), The Theory of Sound: Vol.2, 2nd ed., New York: Dover, 1945.

[13] Nayfeh, A. H., Nonlinear Stability of a Liquid Jet, Phys. Fluids, 1970, vol. 13, no. 4, pp. 841-847.

[14] Wang, F., Tschukin, O., Marques, G. C., Selzer, M., Aghassi-Hagmann, J., and Nestler, B., Breakup of Liquid Jets and the Formation of Satellite and Subsatellite Droplets, arXiv:1805.06818 (2018).

[15] Bazilevskii, A. B. and Rozhkov, A. N., Dynamics of the Capillary Breakup of a Bridge in an Elastic Fluid, Fluid Dyn., 2015, vol. 50, no. 6, pp. 800-811.

[16] Argentina, M., Cohen, A., Bouret, Y., Fraysse, N., and Raufaste, C., One-Dimensional Capillary Jumps, J. Fluid Mech., 2015, VOL. 765, PP.1-16.

[17] Bhagat, R. K., Jha, N. K., Linden, P.F., and Wilson, D., I., On the Origin of the Circular Hydraulic Jump in a Thin Liquid Film, J. Fluid Mech., 2018, vol. 851, R5, 11 pp. 